Japan. J. Math.

Vol. 20, No. 2, 1994

\title{
Zero-one law for a Markov chain
}

\author{
By Hitoshi Mizumachi
}

(Received November 17, 1992)

\section{Introduction}

Let $\mathbf{X}=\left\{X_{n}\right\}_{n=0}^{\infty}$ be an $\mathbf{R}^{d}$-valued random sequence, $\mu$ be the distribution of $\mathbf{X}$ on $\left(\mathbf{R}^{d}\right)^{\infty}$, the space of the sequences in $\mathbf{R}^{d}$, and let $\mu_{X_{n}}$ be the distribution of $X_{n}$. The symbol $\mathcal{B}$ denotes the Borel field of $\left(\mathbf{R}^{d}\right)^{\infty}$ and $\mathcal{B}_{\mu}$ the $\mu$-completion of $\mathcal{B}$, and $\left(\mathbf{R}^{d}\right)_{0}^{\infty}$ denotes the set of sequences in $\mathbf{R}^{d}$ with only finitely many non-zero entries. Define

$$
\left(\mathbf{R}^{d}\right)^{(n)}=\left\{\left(x_{j}\right)_{j=0}^{\infty} \in\left(\mathbf{R}^{d}\right)^{\infty} \mid x_{j}=0 \text { for } j>n\right\}, \quad n \geq 0 .
$$

We denote by $\mathcal{B}_{\infty}$ the tail $\sigma$-field of $\mathbf{X}$, that is, $\mathcal{B}_{\infty}=\bigcap_{n \geq 0} \mathcal{B}_{n}$, where $\mathcal{B}_{n}$ is the smallest $\sigma$-field that makes $X_{n}, X_{n+1}, \ldots$ measurable, and we say $\mathcal{B}_{\infty}$ is trivial if $P(A)=0$ or 1 for all $A \in \mathcal{B}_{\infty}$. It is well known that independent random sequences, random walks and random sequences with strong mixing conditions are tail trivial (see Kolmogorov zero-one law, Hewitt-Savage zero-one law [2] and [4]).

The purpose of this paper is to prove the following :

TheOREM. Let $\mathbf{X}=\left\{X_{n}\right\}_{n=0}^{\infty}$ be an $\mathbf{R}^{d}$-valued Markov chain and assume the following conditions (i), (ii) and (iii).

(i) The tail $\sigma$-field of $\mathbf{X}$ is trivial.

(ii) For every $n$, there exists a $\sigma$-finite Borel measure $m_{n}$ on $\mathbf{R}^{d}$ such that $m_{n}(V)=0$ for every hyperplane $V$ of $\mathbf{R}^{d}$ and that $P\left(X_{n} \in d y \mid X_{n-1}\right)$ is absolutely continuous with respect to $m_{n}$ almost surely.

(iii) $\mu_{X_{0}}(V)=0$ for every hyperplane $V$ of $\mathbf{R}^{d}$. Then we have

$$
\mu(L+\mathbf{a})=0 \text { or } 1 \text { for every affine subspace } L+\mathbf{a} \text { of }\left(\mathbf{R}^{d}\right)^{\infty} .
$$

For an independent random sequence $\mathbf{X}$ with continuous marginal distributions, Hoffmann-Jørgensen [1, Theorem 3.1] proved $\mu(L)=0$ or 1 for every subspace $L$, and this paper extends his result to the case of a Markov chain.

We have proved in [3] that a tail trivial random sequence with absolutely continuous marginal distributions satisfies zero-one law for subgroups, where the 
Lebesgue measure, the Haar measure on $\mathbf{R}^{d}$, plays an essential role. In this paper, by a method different from [3], we prove a zero-one law for affine subspaces of a tail trivial Markov chain when $m_{n}$ is a continuous measure, which may not be the Lebesgue measure.

\section{Proof and corollary}

Proof of Theorem. Let $L$ be a subspace of $\left(\mathbf{R}^{d}\right)^{\infty}, \mathbf{a} \in\left(\mathbf{R}^{d}\right)^{\infty}$ and assume $L+\mathbf{a} \in \mathbf{B}_{\mu}$. Since $\mu$ is a Radon measure, $L+\mathbf{a}$ contains a $\sigma$-compact subset $K$ such that $\mu((L+\mathbf{a}) \backslash K)=0$. Let $L^{\prime}$ be the subspace generated by the $\sigma$-compact set $K-\mathbf{a}$. Then $L^{\prime}$ is $\sigma$-compact, so that $L^{\prime} \in \mathcal{B}$, and we have

$$
K=(K-\mathbf{a})+\mathbf{a} \subset L^{\prime}+\mathbf{a} \subset L+\mathbf{a} \text { and } \mu\left((L+\mathbf{a}) \backslash\left(L^{\prime}+\mathbf{a}\right)\right)=0 .
$$

Therefore we may assume $L+\mathbf{a} \in \mathcal{B}$ without loss of generality. Moreover we may assume $\mathbf{a}=\mathbf{0}$, because $\mathbf{X}-\mathbf{a}=\left\{X_{n}-a_{n}\right\}_{n=0}^{\infty}$ satisfies the assumptions (i), (ii) and (iii).

Let $G$ be the complementary subspace of $\left(\mathbf{R}^{d}\right)_{0}^{\infty} \cap L$ in $\left(\mathbf{R}^{d}\right)_{0}^{\infty}$. Since $G=\bigcup_{n}\left(G \cap\left(\mathbf{R}^{d}\right)^{(n)}\right)$ and each $G \cap\left(\mathbf{R}^{d}\right)^{(n)}$ is $\sigma$-compact, we have

$$
\left(\mathbf{R}^{d}\right)_{0}^{\infty}=\left(\left(\mathbf{R}^{d}\right)_{0}^{\infty} \cap L\right) \oplus G \quad(\text { algebraic direct sum }) \quad \text { and } \quad G \in \mathcal{B} .
$$

Since $(\mathbf{x}, \mathbf{y}) \mapsto \mathbf{x}+\mathbf{y}: L \times G \rightarrow L \oplus G$ is bimeasurable by Theorem 3.9 of [5, page 21], we have $L \oplus G \in \mathcal{B}$, and hence $L \oplus G$ is a tail event, because $\left(\mathbf{R}^{d}\right)_{0}^{\infty} \subset L \oplus G$. Therefore $\mu(L \oplus G)=0$ or 1 ; in particular, we have $\mu(L)=0$ or 1 when $G=\{0\}$. Therefore it is enough to prove $\mu(L)=0$ in case $G \neq\{\mathbf{0}\}$.

Assuming $G \neq\{\mathbf{0}\}$, we define the projections

$$
p: L \oplus G \rightarrow L: \mathbf{x}+\mathbf{y} \mapsto \mathbf{x}, \quad q: L \oplus G \rightarrow G: \mathbf{x}+\mathbf{y} \mapsto \mathbf{y}, \quad(\mathbf{x} \in L, \quad \mathbf{y} \in G),
$$

and

$$
\pi_{j}:\left(\mathbf{R}^{d}\right)^{\infty} \rightarrow \mathbf{R}^{d}:\left(x_{n}\right)_{n=0}^{\infty} \mapsto x_{j}, \quad j \geq 0 .
$$

Then $p$ and $q$ are measurable. Since $G \neq\{\mathbf{0}\}$, we have $q(\mathbf{x}) \neq \mathbf{0}$ for some $\mathbf{x} \in\left(\mathbf{R}^{d}\right)_{0}^{\infty}$. Define

$$
k=\min \left\{n \geq 0 \mid q(\mathbf{x}) \neq \mathbf{0} \text { for some } \mathbf{x} \in\left(\mathbf{R}^{d}\right)^{(n)}\right\},
$$

and choose an $r \geq 0$ such that

$$
\pi_{r} \circ q(\mathbf{x}) \neq 0 \quad \text { for some } \mathbf{x} \in\left(\mathbf{R}^{d}\right)^{(k)} .
$$

Fix such a pair $k, r \geq 0$. Define

$$
\sigma_{k}:\left(x_{j}\right)_{j=0}^{\infty} \mapsto\left(x_{0}, \ldots, x_{k}, 0,0, \ldots\right):\left(\mathbf{R}^{d}\right)^{\infty} \rightarrow\left(\mathbf{R}^{d}\right)^{(k)}
$$


and set $\tau_{k}(\mathbf{x})=\mathbf{x}-\sigma_{k}(\mathbf{x})$. Since $x_{r}=\pi_{r} \circ p(\mathbf{x})=\pi_{r} \circ p\left(\sigma_{k}(\mathbf{x})+\tau_{k}(\mathbf{x})\right)$ for $\mathbf{x} \in L$, we have

$$
\begin{aligned}
\mu(L) & \leq P\left(X_{r}=\pi_{r} \circ p \circ \sigma_{k}(\mathbf{X})+\pi_{r} \circ p \circ \tau_{k}(\mathbf{X})\right) \\
& =\mathbf{E}\left[P\left(X_{r}=\pi_{r} \circ p \circ \sigma_{k}(\mathbf{X})+\pi_{r} \circ p \circ \tau_{k}(\mathbf{X}) \mid X_{k+1}\right)\right] .
\end{aligned}
$$

The Markov property implies

$$
\begin{aligned}
& P\left(X_{r}=\pi_{r} \circ p \circ \sigma_{k}(\mathbf{X})+\pi_{r} \circ p \circ \tau_{k}(\mathbf{X}) \mid X_{k+1}\right) \\
= & \left\{\begin{array}{l}
\int_{\mathbf{R}^{d}} P\left(\pi_{r} \circ p \circ \tau_{k}(\mathbf{X})=z \mid X_{k+1}\right) P\left(X_{r}-\pi_{r} \circ p \circ \sigma_{k}(\mathbf{X}) \in d z \mid X_{k+1}\right) \text { if } r \leq k, \\
\int_{\mathbf{R}^{d}} P\left(X_{r}-\pi_{r} \circ p \circ \tau_{k}(\mathbf{X})=z \mid X_{k+1}\right) P\left(\pi_{r} \circ p \circ \sigma_{k}(\mathbf{X}) \in d z \mid X_{k+1}\right) \text { if } r>k .
\end{array}\right.
\end{aligned}
$$

Assume $r \leq k$ and define linear maps

$$
\begin{gathered}
\varphi:\left(x_{0}, \ldots, x_{k}\right) \mapsto \pi_{r} \circ q\left(x_{0}, \ldots, x_{k}, 0,0, \ldots\right):\left(\mathbf{R}^{d}\right)^{k+1} \rightarrow \mathbf{R}^{d}, \\
\psi: x_{k} \mapsto \varphi\left(0, \ldots, 0, x_{k}\right): \mathbf{R}^{d} \rightarrow \mathbf{R}^{d} .
\end{gathered}
$$

By (1) and the choice of $k, \psi$ is not identically zero, so that $\psi^{-1}\left(x_{k}-\varphi\left(x_{0}, \ldots\right.\right.$, $\left.x_{k-1}, 0\right)$ ) is contained in a hyperplane of $\mathbf{R}^{d}$, and thus we have

$$
m_{k}\left(\psi^{-1}\left(z-\varphi\left(x_{0}, \ldots, x_{k-1}, 0\right)\right)\right)=0 \text { for all } x_{0}, \ldots, x_{k-1}, z \in \mathbf{R}^{d}
$$

Denote by $p_{n}(x, d y)$ the Radon-Nikodym derivative of $P\left(X_{n} \in d y \mid X_{n-1}=x\right)$ with respect to $m_{n}$, and define $m_{0}=\mu_{X_{0}}$,

$$
g(y)=\int_{\left(\mathbf{R}^{d}\right)^{k+1}} p_{1}\left(x_{0}, x_{1}\right) \cdots p_{k+1}\left(x_{k}, y\right) d m_{0}\left(x_{0}\right) \cdots d m_{k}\left(x_{k}\right), \quad y \in \mathbf{R}^{d},
$$

and

$$
h(y)=\left\{\begin{array}{ccc}
g(y)^{-1} & \text { if } & g(y) \neq 0 \\
0 & \text { if } & g(y)=0 .
\end{array}\right.
$$

Since $g(y)$ is the Radon-Nikodym derivative of $\mu_{X_{k+1}}$ with respect to $m_{k+1}$, we have almost surely,

$$
\begin{aligned}
& P\left(\left(\pi_{r}-\pi_{r} \circ p\right) \circ \sigma_{k}(\mathbf{X})=z \mid X_{k+1}\right) \\
& =P\left(\pi_{r} \circ q \circ \sigma_{k}(\mathbf{X})=z \mid X_{k+1}\right) \\
& =P\left(\varphi\left(X_{0}, \ldots, X_{k}\right)=z \mid X_{k+1}\right) \\
& =h\left(X_{k+1}\right) \cdot \int_{\left(\mathbf{R}^{d}\right)^{k+1}} p_{1}\left(x_{0}, x_{1}\right) \cdots p_{k+1}\left(x_{k}, X_{k+1}\right) \\
& \quad \times I_{\left\{z-\varphi\left(x_{0}, \ldots, x_{k-1}, 0\right)\right\}}\left(\psi\left(x_{k}\right)\right) d m_{0}\left(x_{0}\right) \cdots d m_{k}\left(x_{k}\right) \quad \text { for all } z \in \mathbf{R}^{d} .
\end{aligned}
$$


Hence by (2), we have almost surely,

$$
P\left(X_{r}-\pi_{r} \circ p \circ \sigma_{k}(\mathbf{X})=z \mid X_{k+1}\right)=0 \quad \text { for all } z \in \mathbf{R}^{d} .
$$

On the other hand, there exists almost surely a sequence $\left\{z_{j}\right\}_{j=0}^{\infty}$ in $\mathbf{R}^{d}$ such that

$$
P\left(\pi_{r} \circ p \circ \tau_{k}(\mathbf{X})=z \mid X_{k+1}\right)=0 \quad \text { if } z \notin\left\{z_{j}\right\}_{j=0}^{\infty} .
$$

Hence we have almost surely,

$$
\begin{aligned}
& \int_{\mathbf{R}^{d}} P\left(\pi_{r} \circ p \circ \tau_{k}(\mathbf{X})=z \mid X_{k+1}\right) P\left(X_{r}-\pi_{r} \circ p \circ \sigma_{k}(\mathbf{X}) \in d z \mid X_{k+1}\right) \\
= & \sum_{j=0}^{\infty} P\left(\pi_{r} \circ p \circ \tau_{k}(\mathbf{X})=z_{j} \mid X_{k+1}\right) P\left(X_{r}-\pi_{r} \circ p \circ \sigma_{k}(\mathbf{X})=z_{j} \mid X_{k+1}\right)=0,
\end{aligned}
$$

so that

$\mu(L) \leq \mathbf{E}\left[\int_{\mathbf{R}^{d}} P\left(\pi_{r} \circ p \circ \tau_{k}(\mathbf{X})=z \mid X_{k+1}\right) P\left(X_{r}-\pi_{r} \circ p \circ \sigma_{k}(\mathbf{X}) \in d z \mid X_{k+1}\right)\right]=0$.

Next, assume $r>k$. Then by (1), we have $\pi_{r} \circ p(\mathbf{x}) \neq 0$ for some $\mathbf{x} \in$ $\left(\mathbf{R}^{d}\right)^{(k)}$, and $\pi_{r} \circ p(\mathbf{x})=0$ for all $\mathbf{x} \in\left(\mathbf{R}^{d}\right)^{(k-1)}$. Define $\varphi\left(x_{0}, \ldots, x_{k}\right)=\pi_{r} \circ$ $p\left(x_{0}, \ldots, x_{k}, 0,0, \ldots\right)$. Then the proof can proceed similarly as in the case $r \leq k$.

Therefore we have $\mu(L)=0$ when $G \neq\{\mathbf{0}\}$. This completes the proof.

Corollary. Let $\mathbf{X}=\left\{X_{n}\right\}_{n=0}^{\infty}$ be an $\mathbf{R}^{d}$-valued Markov chain as in Theorem, and set $\mu_{X_{0}}=\delta_{b}$ for any fixed $b \in \mathbf{R}^{d}$. Then

$$
\mu(L+\mathbf{a})=0 \text { or } 1 \text { for every affine subspace } L+\mathbf{a} \text { of }\left(\mathbf{R}^{d}\right)^{\infty} .
$$

Proof. As is shown in the proof of Theorem, we may assume $L \in \mathcal{B}$ without loss of generality.

If $(L+\mathbf{a}) \cap \pi_{0}^{-1}(\{b\})=\emptyset$, then $\mu(L+\mathbf{a})=0$. Therefore we may assume that there exists a $\mathbf{v}=\left(v_{n}\right)_{n=0}^{\infty} \in L+\mathbf{a}$ with $v_{0}=b$. Since $L+\mathbf{a}=L+\mathbf{v}$, we have

$$
\begin{aligned}
\left\{\mathbf{X} \in L+\mathbf{a}, X_{0}=b\right\} & =\left\{\left(b, X_{1}, X_{2}, \ldots\right) \in L+\mathbf{v}\right\} \\
& =\left\{\left(0, X_{1}, X_{2}, \ldots\right) \in L+\mathbf{v}-(b, 0,0, \ldots)\right\} \\
& =\left\{\left(0, X_{1}, X_{2}, \ldots\right) \in L+\left(0, v_{1}, v_{2}, \ldots\right)\right\} \\
& =\left\{\left(X_{1}, X_{2}, \ldots\right) \in L_{0}+\left(v_{1}, v_{2}, \ldots\right)\right\}
\end{aligned}
$$

where $L_{0}=\left\{\left(x_{j}\right)_{j=1}^{\infty} \in\left(\mathbf{R}^{d}\right)^{\infty} \mid\left(0, x_{1}, x_{2}, \ldots\right) \in L\right\}$. Then $L_{0} \in \mathcal{B}$ and $\left(X_{1}, X_{2}, \ldots\right)$ satisfies all the assumptions of Theorem. Hence we have $\mu(L+\mathbf{a})=0$ or 1 . 
ExAmPle. Let $\mathbf{X}=\left\{X_{n}\right\}_{n=0}^{\infty}$ be a real Markov chain, and assume that the tail $\sigma$-field of $\mathbf{X}$ is trivial, every transition probability is absolutely continuous with respect to the Lebesgue measure and the initial distribution is either a continuous measure or a unit measure at a point. Then the distribution $\mu$ of $\mathbf{X}$ satisfies the zero-one law for affine subspaces.

Acknowledgement. The author wishes to express his gratitude to Professor $\mathrm{H}$. Sato for his valuable advice and encouragement.

\title{
References
}

[ 1 ] J. Hoffmann-Jørgensen, Integrability of seminorms, the 0-1 law and the affine kernel for product measures, Studia Math., 61 (1977), 137-159.

[ 2 ] M. Loève, Probability Theory I, 4th ed., Springer, New York Berlin Heidelberg, 1977.

[ 3 ] H. Mizumachi and H. Sato, Continuity of quasi-invariant measures and zero-one laws on groups, J. Functional Analysis, 120(1) (1994), 188-200.

[ 4 ] S. Orey, Limit theorems for Markov chain transition probabilities, Van Nostrand, 1971.

[ 5 ] K. R. Parthasarathy, Probability measures on metric spaces, Academic Press, New York San Francisco London, 1967.

\author{
Department of Mathematics \\ KYUSHU UNIVERSITY-33 \\ HAKOZAKI, FUKUOKA 812, JAPAN \\ Present Address \\ KUMAMOTO UNIVERSITY \\ College of Medical Science \\ KuHONJi, Kumamoto 862, JAPAN
}

